\title{
Compensation of domain modelling errors in the inverse source problem of the Poisson equation: application in electroencephalographic imaging
}

\author{
Alexandra Koulouri ${ }^{\mathrm{a}}$, Ville Rimpiläinen ${ }^{\mathrm{b}, *}$, Mike Brookes $^{\mathrm{a}}$, Jari P. \\ Kaipio $^{\mathrm{b}, \mathrm{c}, \mathrm{d}}$ \\ ${ }^{a}$ Department of Electrical and Electronic Engineering, Imperial College London, \\ Exhibition Road, London SW7 2BT, United Kingdom \\ ${ }^{b}$ Department of Mathematics, University of Auckland, Private bag 92019, Auckland \\ 1142, New Zealand \\ ${ }^{c}$ Department of Applied Physics, University of Eastern Finland, FI-70211 Kuopio, \\ Finland \\ ${ }^{d}$ The Dodd-Walls Centre for Photonic and Quantum Technologies, Dunedin 9016, New \\ Zealand
}

\begin{abstract}
In the inverse source problem of the Poisson equation, measurements on the domain boundaries are used to reconstruct sources inside the domain. The problem is an ill-posed inverse problem and it is sensitive to modelling errors of the domain. These errors can be boundary, structure and material property errors, for example. In this paper, we investigate whether the recently proposed Bayesian approximation error (BAE) approach could be used to alleviate the source estimation errors when an approximate model for the domain is employed. The BAE is based on postulating a probabilistic model for the uncertainties, in this case the geometry and structure of the domain, and to carry out approximate marginalization over these nuisance parameters. We particularly consider electroencephalography (EEG) source imaging as an application. EEG is a diagnostic brain imaging modality, and it can be used to reconstruct neural sources in the brain from electric potential measurements along the scalp. In the feasibility study, we assess to which degree one can recover from the modelling errors that are induced by
\end{abstract}

\footnotetext{
${ }^{*}$ Corresponding author. Tel.: +64 99238818

Email address: vrimpila@gmail.com (Ville Rimpiläinen)
} 
the use of the three concentric circle head model instead of an anatomically accurate head model. The studied domain modelling errors include errors in the geometry of the exterior boundary and the structure of the interior. We show that, in particular with superficial dipole sources, the BAE yields estimates that can in some cases be considered adequately accurate. This would avoid the need for the extraction of the accurate head features which is conventionally carried out via expensive and time consuming auxiliary imaging modalities such as magnetic resonance imaging.

Keywords: Inverse source problem, Poisson equation, electroencephalography, Bayesian inversion, modelling errors

\section{Introduction}

In the inverse source problem of the Poisson equation, the goal is to reconstruct sources inside the computational domain based on measurements around its boundaries. The problem has many practical applications such as bioluminescence tomography [14], and electroencephalographic [12] and magnetoencephalographic imaging [13]. The inverse source problem is an ill-posed inverse problem, and stable estimates cannot be computed from noisy boundary measurements without either regularization or by employing prior models. Moreover, the inverse solution is highly sensitive to modelling errors in addition to measurement errors. Typical modelling errors are related to the geometry, internal structure and material properties of the domain. Because such errors are commonly encountered in brain imaging, we consider electroencephalography (EEG) source imaging as a potential application in this paper.

EEG is a routinely used brain imaging modality to diagnose, for example, epilepsy. In EEG source problem, neural sources are reconstructed inside the brain based on electric potential measurements around the scalp, and it is well known that the inverse solution depends strongly on the accuracy of discretized head geometry $[2,4,7,8,16,39,49,54]$ and the accuracy of electric conductivity modelling of different tissues [3, 47, 25, 48, 34, 50, 51]. The head features can be extracted, to some extent, by using multi-modal imaging (computed tomography / diffusion magnetic resonance imaging), for example. However, such imaging is an expensive task and requires robust 
image segmentation, registration and post-processing algorithms [42]. Therefore, approaches that allowed the use of approximate head models would be highly desirable.

In this paper, we investigate to which extent the domain modelling errors can be compensated by considering the Bayesian approximation error (BAE) approach which was introduced in [19]. BAE is applied solely to the discretized problem with finite number of boundary measurements, and the idea is to use an approximate model for the domain and to estimate the statistics of the errors between the approximate and accurate models. These errors are induced by a postulated probabilistic model for the uncertainties, in this case, the domain geometry and structure. In the resulting overall observation model, the modelling errors appear as additive errors which are then marginalized using a Gaussian approximation.

Previously, BAE has been shown to be a versatile and efficient approach to compensate for various approximation and modelling errors, for example, in electrical impedance tomography (EIT) $[24,26,30,31]$ and diffuse optical tomography (DOT) $[1,22,23,29,44,45]$. In particular, EIT was employed for the reconstruction of the conductivity distributions in the thorax, and the approximation errors related to the use of a generic geometry (cylinder) instead of an actual thorax with severe model reduction were handled with BAE in [31]. Similarly in DOT, the effects of mismodelled head geometry were compensated with BAE in [29]. Thus, the BAE has been shown to be successful in compensating errors related to the geometric mismodelling in diffuse tomographic problems.

In this paper, in contrast to diffuse tomographic problems, we are not interested in the material properties of the domain but a vector-valued source field that, however, depends on the modelling of the domain. In addition to boundary mismodelling, we also consider modelling errors related to the interior of the domain which was not done in [31, 29]. In the EEG source imaging examples, we consider only focal dipole sources, that are common in epilepsy, and the case of finite number of boundary measurements (instead of continuous boundary data). In order to recover focal sources, we employ sparsity promoting priors rather than Gaussian smoothing priors as in [31, $29]$.

We use an anatomical atlas to construct the model for the distribution 
of the head geometry and structure. Because the head can be segmented into various number of different tissues, we consider here the three and five compartment models as the anatomically accurate domain models (head conductivity models). Then the three concentric circle model is used as the approximate head model. To access the ground truth, we use simulations in this feasibility study. We also consider both noiseless and noisy data to isolate the effects of domain modelling errors, and to assess the practical case in which both modelling and measurement errors are present. When successful, the use of BAE would thus allow the same generic head model to be used for all patients.

\section{Theory}

In this section, we define the forward models used in this paper, give a brief review of the Bayesian framework for inverse problems, and the Bayesian approximation error approach. More information about the Bayesian framework in general can be found in, for example, $[6,19,43]$ and the approximation error approach in particular in $[1,19,18,17]$.

\subsection{Poisson equation}

The computational domain is denoted with $\Omega$ and its material properties with $\sigma(x)$ where $x \in \Omega$. The Poisson equation has the form

$$
\nabla \cdot \sigma(x) \nabla u(x)=f(x), \quad x \in \Omega
$$

where $u$ is the scalar potential and $f(x)$ is the source term. The boundary conditions are

$$
\begin{aligned}
u & =0, \quad x \in S_{\mathrm{D}} \\
\sigma(x) \frac{\partial u}{\partial \hat{n}} & =0, \quad x \in S_{\mathrm{N}},
\end{aligned}
$$

where $\partial \Omega=S_{\mathrm{D}} \cup S_{\mathrm{N}}$ is the boundary, $S_{\mathrm{D}} \cap S_{\mathrm{N}}=\varnothing$, and $\hat{n}$ is the unit normal vector of the boundary.

In case of EEG imaging, the Poisson equation can be used under the quasistatic approximation of the Maxwell's equations. Furthermore, in EEG the 
source term is often of the form $f(x)=\nabla \cdot d(x)$ where $d(x): \Omega \mapsto \mathbb{R}^{k}$ is a vector valued function that describes the neural sources as idealized electric dipoles [41]. Here, $k$ is equal to either 2 or 3 depending whether the analysis is carried out in $2 \mathrm{D}$ or $3 \mathrm{D}$.

\subsection{Forward models}

The mapping from the source field to the $i$ th boundary measurement, $v_{i}=u\left(x_{i}\right)$ where $x_{i} \in S_{\mathrm{N}}$, can be written as

$$
v_{i}=\int_{\Omega} a\left(\lambda, \sigma, x_{i}, x\right) \cdot d(x) \mathrm{d} x,
$$

where $a\left(\lambda, \sigma, x_{i}, x\right)$ is a vector-valued function, that depends on the parameterizations of the geometry $\lambda$ and material properties $\sigma$ of the domain [33].

For a finite set of boundary measurements, we write the observation model as

$$
v=A(\lambda, \sigma) d+e,
$$

where $v \in \mathbb{R}^{m}, m$ is the number of measurements, $A$ is the mapping from the source configuration to all the measurements and $e$ is the measurement noise. In case of EEG imaging, the mapping $A(\lambda, \sigma)$ is called the lead field [12]. It is available in a closed form only for few simple cases, such as concentric spheres [53, 55], and in more general cases it is approximated numerically $[11,32,52]$.

For numerical implementations, the domain is discretized and the observation model is written as

$$
v=A_{h}(\lambda, \sigma) d+e,
$$

where $h$ denotes the discretization level, $A_{h}(\lambda, \sigma) \in \mathbb{R}^{m \times k n}$ is the lead field matrix, and $d \in \mathbb{R}^{k n}$ is the distributed dipole source configuration. In this paper, $d$ and $e$ are considered mutually (statistically) independent.

We define the accurate numerical model

$$
v=A_{\delta}(\lambda, \sigma) \bar{d}+e
$$


in which $(\lambda, \sigma)$ are known and the numerical approximation $A_{\delta}$ for $A$ is accurate in the sense that the numerical (discretization related) errors of the predictions are smaller than measurement errors. See Section 2.4 for the exact definition. The notation $\bar{d}$ refers to a numerically accurate representation of $d$.

In BAE, we replace the accurate model, $A_{\delta}$, with an approximating computational model $A_{0} \in \mathbb{R}^{m \times k n_{0}}$ when the (inverse) estimates are computed. Moreover, in the approximate model, we employ nominal fixed values $\left(\lambda_{0}, \sigma_{0}\right)$ for the uninteresting unknowns (nuisance parameters). We can write

$$
\begin{aligned}
v & =A_{0}\left(\lambda_{0}, \sigma_{0}\right) d+\left(A_{\delta}(\lambda, \sigma) \bar{d}-A_{0}\left(\lambda_{0}, \sigma_{0}\right) d\right)+e \\
& =A_{0}\left(\lambda_{0}, \sigma_{0}\right) d+\varepsilon+e \\
& =A_{0}\left(\lambda_{0}, \sigma_{0}\right) d+\nu
\end{aligned}
$$

where $\varepsilon=A_{\delta}(\lambda, \sigma) \bar{d}-A_{0}\left(\lambda_{0}, \sigma_{0}\right) d$ is the approximation error induced by using the approximate model and $\nu=\varepsilon+e$. The approximation error is thus a random variable (vector) whose distribution is determined by the joint (prior) distribution $\pi(\lambda, \sigma, d)$. Assuming that measurement errors are mutually independent with $(\lambda, \sigma, d), e$ and $\varepsilon$ are also mutually independent. The quantities $\bar{d}$ and $d$ are related according to $d=P \bar{d}$ where $P: \mathbb{R}^{k n_{\delta}} \rightarrow$ $\mathbb{R}^{k n_{0}}$ is a linear model reduction map (a projection) from the fine domain to the coarse approximative domain.

\subsection{Bayesian framework}

In the Bayesian framework, all model variables are considered as random variables. The modelling of joint distribution of these variables is based on the observation model and a model for the prior distribution of the unknowns. All inference on the (posterior) uncertainty of the interesting variable(s) is based on the posterior density model that can be written with the help of the Bayes' formula as

$$
\pi(d \mid v)=\frac{\pi(v \mid d) \pi(d)}{\pi(v)} \propto \pi(v \mid d) \pi(d)
$$

where $\pi(v \mid d)$ is the likelihood, $\pi(d)$ the prior and $\pi(v)$ is used for normalization. Thus, in order to get the posterior, we need to determine the likelihood first. 
In approximation error approach, the likelihood can be derived with the help of the joint density $\pi(v, \nu, d)=\pi(v, \nu \mid d) \pi(d)=\pi(v \mid \nu, d) \pi(\nu \mid d) \pi(d)$ as

$$
\pi(v \mid d)=\int \pi(v, \nu \mid d) \mathrm{d} \nu=\int \pi(v \mid \nu, d) \pi(\nu \mid d) \mathrm{d} \nu .
$$

Note that all uncertainty in the nuisance parameters $(\lambda, \sigma)$ is embedded in the joint distribution $\pi(d, \varepsilon)$. For details of the formal derivation of the likelihood, see, for example [17].

Based on (10), the conditional density of the measurements given the source field can be written as

$$
\pi(v \mid d, \nu)=\delta\left(v-A_{0}\left(\lambda_{0}, \sigma_{0}\right) d-\nu\right),
$$

where $\delta(\cdot)$ is the Dirac delta distribution.

The likelihood thus can be formulated through a convolution operation as

$$
\begin{aligned}
\pi(v \mid d) & =\int \delta\left(v-A_{0}\left(\lambda_{0}, \sigma_{0}\right) d-\nu\right) \pi(\nu \mid d) \mathrm{d} \nu \\
& =\pi_{\nu \mid d}\left(v-A_{0}\left(\lambda_{0}, \sigma_{0}\right) d \mid d\right)
\end{aligned}
$$

where the subscript $\nu \mid d$ is used to clarify that the probability density is that of $\nu$ given $d$.

In BAE, where efficient computational implementations are of consideration, $\pi_{e}$ and $\pi_{\varepsilon \mid d}$ are approximated with Gaussian distributions $e \sim \mathcal{N}\left(e_{*}, \Gamma_{e}\right)$ and $\varepsilon_{\mid d} \sim \mathcal{N}\left(\varepsilon_{* \mid d}, \Gamma_{\varepsilon \mid d}\right)$, respectively. The approximate likelihood is then Gaussian (in our case)

$$
\begin{aligned}
& \tilde{\pi}_{\nu \mid d}(v-\left.A_{0}\left(\lambda_{0}, \sigma_{0}\right) d \mid d\right) \propto \\
& \exp \left(-\frac{1}{2}\left(v-A_{0}\left(\lambda_{0}, \sigma_{0}\right) d-\nu_{* \mid d}\right)^{\mathrm{T}}\right. \\
&\left.\Gamma_{\nu \mid d}^{-1}\left(v-A_{0}\left(\lambda_{0}, \sigma_{0}\right) d-\nu_{* \mid d}\right)\right)
\end{aligned}
$$

with $\nu_{* \mid d}=\varepsilon_{* \mid d}+e_{*}$ and $\Gamma_{\nu \mid d}=\Gamma_{\varepsilon \mid d}+\Gamma_{e}$.

The Gaussian approximation of the posterior density (11) is thus of the form

$$
\left.\tilde{\pi}(d \mid v) \propto \tilde{\pi}_{\nu \mid d}\left(v-A_{0}\left(\lambda_{0}, \sigma_{0}\right) d \mid d\right)\right) \pi(d) .
$$


Thus, for the determination of the posterior model $\tilde{\pi}(d \mid v)$, we need to postulate a prior model $\pi(d)$ first and compute the related (induced) conditional mean $\varepsilon_{* \mid d}$ and conditional covariance $\Gamma_{\varepsilon \mid d}$.

\subsection{Approximation error statistics}

Define the (stacked) variable $z$ as

$$
z=\left(\begin{array}{l}
d \\
\varepsilon
\end{array}\right) .
$$

In BAE, the joint density of $d$ and $\varepsilon$ is approximated as the Gaussian $z \sim$ $\mathcal{N}\left(z_{*}, \Gamma_{z}\right)$

$$
\tilde{\pi}(z) \propto \exp \left(-\frac{1}{2}\left(z-z_{*}\right)^{T} \Gamma_{z}^{-1}\left(z-z_{*}\right)\right)
$$

where the mean and covariance are

$$
\begin{aligned}
& z_{*}=\left[\begin{array}{l}
d_{*} \\
\varepsilon_{*}
\end{array}\right] \\
& \Gamma_{z}=\left[\begin{array}{ll}
\Gamma_{d} & \Gamma_{d \varepsilon} \\
\Gamma_{\varepsilon d} & \Gamma_{\varepsilon}
\end{array}\right] .
\end{aligned}
$$

The respective conditional mean and covariance of the error given the source field can then be written as $[10,19]$

$$
\begin{aligned}
\varepsilon_{* \mid d} & =\varepsilon_{*}+\Gamma_{\varepsilon d} \Gamma_{d}^{-1}\left(d-d_{*}\right) \\
\Gamma_{\varepsilon \mid d} & =\Gamma_{\varepsilon}-\Gamma_{\varepsilon d} \Gamma_{d}^{-1} \Gamma_{d \varepsilon} .
\end{aligned}
$$

In practice, these are approximated using a Monte Carlo simulation from the overall prior model $\pi(\lambda, \sigma, d)$ and using the respective sample mean and covariance, see Section 3.2.

When $\operatorname{var}\left(e_{k}\right) \geq c\left(\varepsilon_{*, k}^{2}+\operatorname{var}\left(\varepsilon_{k}\right)\right)$, where $c \geq 2$, we say that the measurement errors dominate the approximation errors [17]. In such a case (assuming zero mean measurement errors), we also have

$$
\mathbb{E}\left(\|e\|^{2}\right)=\operatorname{tr} \Gamma_{e} \geq c\left(\left\|\varepsilon_{*}\right\|^{2}+\operatorname{tr} \Gamma_{\varepsilon}\right)
$$

where $\operatorname{tr}(\cdot)$ denotes the trace of a matrix. 


\section{5. $L_{1,2}$ (group lasso) prior model with weight factors}

In this paper, we consider sparse focal source models and we employ an $L_{1,2}$ norm induced prior model. The $L_{1,2}$ norm is defined as the $L_{1}$-norm of the source strengths. The prior distribution $\pi(d)$ is thus of the form

$$
\pi(d) \propto \exp \left(-\frac{\alpha}{2} \sum_{i=1}^{n_{0}} w_{i}\left\|d_{i}\right\|_{2}\right)
$$

where $d_{i}=\left(d_{i x}, d_{i y}\right)$ and $\left\|d_{i}\right\|_{2}=\sqrt{d_{i x}^{2}+d_{i y}^{2}}$ is the strength of the source at location $i, \alpha$ is a scaling constant that fixes the distribution of the source strengths, and $w_{i}$ is a depth weighting factor. The $L_{1,2}$ norm has previously been used, for example, in $[9,15,46]$ and it effectively makes the source distribution focal.

In addition, we employ depth weighting factors $w_{i}$ to reduce the bias of the $L_{1,2}$ based prior favoring superficial source distributions. In this paper, the weights were selected similarly as in [15], where they were extracted from the covariance matrix of the minimum-norm solution of the source imaging problem, that is, $\left[w_{1}, \ldots, w_{n 0}\right]=\operatorname{diag}\left(A_{0}^{\mathrm{T}}\left(A_{0} A_{0}^{\mathrm{T}}\right)^{-1} A_{0}\right)$. For alternative choices of weighting factors, see $[21,36,46]$.

\subsection{Maximum a posteriori estimate}

Carrying out general statistical inference from the posterior density in a high-dimensional parameter space is in most cases not practical since such inference calls for carrying out a Markov chain Monte Carlo run. In this paper, we compute only maximum a posteriori (MAP) estimates. The MAP estimate of $d$ is

$$
\hat{d}=\arg \max _{d} \tilde{\pi}(d \mid v) .
$$

With the help of $(17), \hat{d}$ can be estimated by minimizing the exponential

$$
\hat{d}=\min _{d}\left\|L_{\nu}\left(v-A_{0}\left(\lambda_{0}, \sigma_{0}\right) d-\nu_{* \mid d}\right)\right\|_{2}^{2}+\alpha \sum_{i=1}^{n_{0}} w_{i}\left\|d_{i}\right\|_{2}
$$

where $L_{\nu}$ comes from the Cholesky factorization $\Gamma_{\nu \mid d}^{-1}=L_{\nu}^{T} L_{\nu}$. In this paper, we solve the resulting non-linear convex minimization problem using the truncated Newton interior point method [5, 20]. 


\section{Numerical simulations}

As an application, we study the use of BAE for the compensation of head geometry and structure errors in EEG source imaging. For the BAE statistics, we need a distribution for the head geometry and structure. In this section, we discuss the anatomical atlas which is used for an ensemble of accurate domain models, the computation of the approximation error statistics, and the test cases. As the approximating domain model $A_{0}\left(\lambda_{0}, \sigma_{0}\right)$, we employ the three concentric circle model [12]. Below, we refer to this as the standard model.

\subsection{Domain models}

For this study, magnetic resonance imaging (MRI) data from the OASIS project database [28] were used to build a training set of 32 accurate head models. The analysis was carried out in 2D, and a transverse (axial) cross section above the eyes was segmented for the analysis. The scalp and different brain compartments, i.e. cerebrospinal fluid (CSF), gray matter (GM) and white matter (WM), were segmented using FieldTrip toolbox [35], and threshold and morphology operators were used for skull segmentation [38]. The electric conductivities were taken to be $0.33 \mathrm{~S} / \mathrm{m}$ for scalp, $0.016 \mathrm{~S} / \mathrm{m}$ for skull, $1.76 \mathrm{~S} / \mathrm{m}$ for CSF, 0.14 for GM and $0.33 \mathrm{~S} / \mathrm{m}$ for WM [33].

Then 32 measurement locations and a fixed ground point (above the righthand-side ear) were uniformly spaced around the domain. An example of the segmentation and the electrode configuration is shown in Figure 1. Finally, the domains were discretized using triangular elements, and the corresponding lead field matrices were computed using a finite element method with linear nodal basis functions [52].

\subsection{Computation of the approximation error statistics}

In BAE, an ensemble of model predictions using the accurate model and sampled unknowns is needed. It is not essential that analytical expressions for the related densities are available, thus, samples from, say, an anatomical atlas are adequate. These predictions by the accurate model are computed off-line and the accurate model is never needed for inversion. When some of 
the unknowns are modelled as mutually independent, Cartesian product of the sample sets are typically constructed. As a result, one has the model predictions computed over the prior distribution $\left\{A_{\delta}\left(\lambda^{(j)}, \bar{\sigma}_{0}\right) \bar{d}^{(j)}, j=1, \ldots, J\right\}$ as well as the respective predictions $\left\{A_{0}\left(\lambda_{0}, \sigma_{0}\right) d^{(j)}, j=1, \ldots, J\right\}$. In this paper, the following procedure was used to construct the sample sets

1. First, one of the domain geometries, $\lambda^{(j)}$, was randomly chosen. The corresponding lead field matrix is denoted as $A_{\delta}\left(\lambda^{(j)}, \bar{\sigma}_{0}\right)$ where $\bar{\sigma}_{0}$ refers to the accurate (either 3 or 5 compartment) domain structure.

2. A random source field, $\bar{d}^{(j)}$, was generated.

3. The generated source field was projected onto the coarse grid of the standard model, $d^{(j)}=P^{(j)} \bar{d}^{(j)}$.

4. The corresponding approximation error was computed, $\varepsilon^{(j)}=A_{\delta}\left(\lambda^{(j)}, \bar{\sigma}_{0}\right) \bar{d}^{(j)}-$ $A_{0}\left(\lambda_{0}, \sigma_{0}\right) d^{(j)}$, where $A_{0}\left(\lambda_{0}, \sigma_{0}\right)$ is the lead field matrix of the standard model.

The procedure was repeated $J=160000$ times, and the sample-based approximations for the error statistics were computed as

$$
\begin{aligned}
\varepsilon_{*} & =\frac{1}{J} \sum_{j=1}^{J} \varepsilon^{(j)} \\
\Gamma_{\varepsilon} & =\frac{1}{J-1} \sum_{j=1}^{J}\left(\varepsilon^{(j)}-\varepsilon_{*}\right)\left(\varepsilon^{(j)}-\varepsilon_{*}\right)^{\mathrm{T}} .
\end{aligned}
$$

\subsection{Simulated test cases}

For the evaluation, a domain that was not included in the training set of domain models was first selected. As test cases, we considered different sparse dipole source fields with either one or three focal sources in the grey matter. The $x$ - and $y$-components of the dipole moments were drawn from Gaussian distributions resulting in chi-squared (with two degrees of freedom) distribution for the dipole strength. The spatial dipole source fields were then smoothed using a Gaussian $5 \times 5$ low pass filter to simulate sets of pyramidal neurons with similar orientation that commonly give rise to the EEG measurements. Then, the boundary data were computed using the accurate domain model, $A_{\delta}$. For all these data, three different reconstructions were computed and evaluated as explained in the next section. 


\subsection{Different reconstructions and comparison metrics}

Three different estimates were computed for all simulated measurements. The first ones were computed using a coarsely discretized version $A_{c}$ of the accurate model. The coarse version of the lead field matrix was used instead of $A_{\delta}$ in order to avoid the so-called inverse crime, which would result in grossly overoptimistic results [19]. The second estimates were computed using the standard model $A_{0}$ for the domain that consisted of three concentric circles [12] representing, respectively, the scalp, skull and brain and using the WM conductivity for the brain region. The third estimates were computed using the standard model but now incorporating the approximation errors statistics.

The different maximum a posteriori (MAP) estimates were used for the evaluation and visualization of the results. The MAP estimate in the accurate domain is

$$
\hat{d}_{\mathrm{acc}}=\min _{d}\left\|L_{e}\left(v-A_{c}(\lambda, \sigma) d\right)\right\|_{2}^{2}+\alpha_{1} \sum_{i=1}^{n_{\mathrm{acc}}} w_{i}\left\|d_{i}\right\|_{2},
$$

where $L_{e}$ is given by the Cholesky factorization $\Gamma_{e}^{-1}=L_{e}^{\mathrm{T}} L_{e}$, and $A_{c}(\lambda, \sigma)$ refers to the lead field matrix that is evaluated in the coarsely discretized domain with the correct geometry $\lambda$ and structure (conductivity modelling) $\sigma$.

The MAP estimate in the standard geometry is

$$
\hat{d}_{\mathrm{stan}}=\min _{d}\left\|L_{e}\left(v-A_{0}\left(\lambda_{0}, \sigma_{0}\right) d\right)\right\|_{2}^{2}+\alpha_{2} \sum_{i=1}^{n_{0}} w_{i}\left\|d_{i}\right\|_{2} .
$$

The MAP estimate that utilizes the approximation error approach in the standard geometry is

$$
\hat{d}_{\mathrm{BAE}}=\min _{d}\left\|L_{\nu}\left(v-A_{0}\left(\lambda_{0}, \sigma_{0}\right) d-\nu_{* \mid d}\right)\right\|_{2}^{2}+\alpha_{3} \sum_{i=1}^{n_{0}} w_{i}\left\|d_{i}\right\|_{2} .
$$

The scaling coefficients were chosen so that the prior variance of the source strengths is approximately the variance of the strengths of the simulated ones. 
The values of $\alpha_{1}, \alpha_{2}$ and $\alpha_{3}$ were defined as fractions of the corresponding maximum value $\alpha_{\max }$ which can be derived based on [20] and in this case has the form

$$
\alpha_{\max }=\max \left(\frac{2\left(\left(v^{\mathrm{T}} A_{x}^{i}\right)^{2}+\left(v^{\mathrm{T}} A_{y}^{i}\right)^{2}\right)^{1 / 2}}{w_{i}}\right),
$$

where $i=1, \ldots, n$ is the node index, $v$ are the measured boundary potentials, $A_{x}$ and $A_{y}$ are the $x$ and $y$ partitions of the $i$ th lead field vector, and $w_{i}$ is the weight factor. The used scaling coefficients and details of the meshes are given in Table 1.

Table 1: Finite element meshes and scaling coefficients.

\begin{tabular}{cccc} 
Mesh & $\begin{array}{c}\text { No. of } \\
\text { nodes }\end{array}$ & $\begin{array}{c}\text { No. of } \\
\text { elements }\end{array}$ & $\begin{array}{c}\alpha_{1,2,3} \text { (fraction } \\
\text { of } \alpha_{\max } \text { ) }\end{array}$ \\
\hline Dense accurate & 2342 & 4549 & - \\
Coarse accurate & 1236 & 2337 & $0.0005-0.01$ \\
Concentric (standard) & 728 & 1387 & $0.001-0.05$ \\
Concentric (BAE) & 728 & 1387 & $0.05-0.10$ \\
\hline
\end{tabular}

For the quantification of the reconstruction errors, we employ the socalled earth mover's distance (EMD). EMD is defined as the minimum cost that must be done to transform one normalized discrete signal into the other given the metric between the discrete points of the domain [40,37]. EMD has previously been used in the context of EEG imaging in [15, 27] since it is suitable for comparing images with possibly non-overlapping support such as sparse vectors.

\section{Results and discussion}

Two different types of test cases were studied. In the first set of test cases the aim is to isolate the domain geometry related error and its effect from the measurement errors. In the second set of test cases, domain structure (conductivity modelling) and measurement errors were considered in addition to the geometry related errors. 


\subsection{Compensation for domain geometry errors only, three compartment for- ward model}

In the first set of test cases, only the effects of the unknown geometry were studied. A domain that was not included in the training domain models and different source fields were selected. The simulated measurements were computed using the forward model with accurate geometry and three compartment structure (scalp, skull, brain as shown in Figure 1). The conductivities of the different compartments were $0.33,0.016$ and $0.33 \mathrm{~S} / \mathrm{m}$, respectively.

Four test cases with a single active focal region are shown Figure 2. The accurate model gives the lowest EMD values as expected. Also visually, the estimates are good. Note that there are still numerical model reduction errors present since the lead field matrices $A_{\delta}$ and $A_{c}$ are not the same. Regarding the estimates that employ the approximate forward model, BAE is able to localize the dipole sources more accurately than the standard model. This is clear from the reconstruction both visually and with respect to the EMD values. It is noted, that the differences between the EMD values given by the accurate and the BAE model are relatively small. The most apparent feature of the standard estimates is that the superficial dipole sources (cases A-C) are located systematically too deep.

Two test cases with three focal sources are shown in Figure 3. In the first case, the BAE gives a good estimate for the source locations in terms of the EMD and also visually. In this case, all sources are relatively superficial. In the second case, two sources are superficial and the third source is a deep one. In this case, the BAE estimates for the superficial sources are relatively good while the deep source is mislocated. On the other hand, the standard estimate seems to group the two sources into one, and the EMD values are higher than with the BAE estimates.

4.2. Compensation of domain geometry and structure errors, with measurement errors, five compartment forward model

In these test cases, we assess the use of BAE when the domain geometry

is unknown, the domain structure (conductivity modelling) is not accurate and the boundary measurements are noisy. The measurement data was now 
computed using the accurate five compartment structure (scalp, skull, CSF, GM and WM as shown in Figure 1). Random white noise was simulated and added to the measurements with signal to noise ratios of 40 and $20 \mathrm{~dB}$. Moreover, rather than computing estimates for a single draw from the distribution of the noise process, we compute an estimate for the distribution of the reconstructed dipole sources over the distribution of the additive noise. This was carried out by computing 30 different realizations of the noise process.

In the reference, or the accurate solution, the accurate domain geometry and precise structure are known. In the standard and the BAE estimates, the domain consists of only three circular compartments (scalp, skull, brain with the conductivity values $0.33,0.016$ and $0.33 \mathrm{~S} / \mathrm{m}$, respectively) which induces a domain structure error in addition to the geometrical error.

Two test cases with a single focal source were selected, these correspond to the cases (C) and (D) in Figure 2. Figure 4 shows the average of the 30 reconstructions, as well as the average and standard deviation of the corresponding EMD values. The figures are now to be interpreted as statistical distributions of the solutions (over the distribution of the noise). With respect to the EMD values, the BAE estimates are clearly better than the standard estimates, in particular, in the case of the superficial source case. Based on the EMD values, the BAE solutions have the highest standard deviations which is to be expected: it can be shown analytically that the posterior distribution of the BAE posterior model always has larger variances than the standard model.

A test case with three equal strength sources is shown in Figure 5. This case gives the largest difference between the reference and BAE estimates. The latter, however is able to locate three different sources (in most realizations) in the $40 \mathrm{~dB}$ noise case, while the standard model finds only two sources, one in each hemisphere. With respect to the EMD values, the BAE estimates are better than standard estimates in both $40 \mathrm{~dB}$ and $20 \mathrm{~dB}$ noise cases.

\subsection{Distribution of EMD values over randomized source distributions}

To evaluated the performance of the models, 500 dipole source distributions were simulated and the corresponding EMD values were computed. 
The results are shown in Table 2 with the corresponding domain structure, number of sources and SNR level. For all cases, the accurate model and BAE resulted in similar EMD values. The EMD values for the standard estimates are higher throughout, except for the high noise level cases.

Table 2: Means and standard deviations of EMD values over 500 simulated dipole distributions.

\begin{tabular}{cccccc}
$\begin{array}{c}\text { No. of domain } \\
\text { compartments }\end{array}$ & $\begin{array}{c}\text { No. of } \\
\text { sources }\end{array}$ & $\begin{array}{c}\text { SNR } \\
(\mathrm{dB})\end{array}$ & $\begin{array}{c}\text { EMD: } \\
\text { accurate }\end{array}$ & $\begin{array}{c}\text { EMD: } \\
\text { standard }\end{array}$ & $\begin{array}{c}\text { EMD: } \\
\text { BAE }\end{array}$ \\
\hline 3 & 1 & $\infty$ & $5.9 \pm 3.9$ & $12.8 \pm 4.5$ & $7.6 \pm 3.6$ \\
3 & 3 & $\infty$ & $16.9 \pm 6.1$ & $21.3 \pm 5.7$ & $17.3 \pm 6.4$ \\
5 & 1 & 40 & $11.6 \pm 4.8$ & $15.8 \pm 4.5$ & $11.5 \pm 4.7$ \\
5 & 1 & 20 & $16.9 \pm 6.2$ & $16.2 \pm 4.7$ & $14.4 \pm 6.1$ \\
5 & 3 & 40 & $19.5 \pm 5.3$ & $23.2 \pm 7.0$ & $19.2 \pm 5.0$ \\
5 & 3 & 20 & $23.3 \pm 5.3$ & $26.9 \pm 7.0$ & $22.6 \pm 5.4$ \\
\hline
\end{tabular}

\section{Conclusions}

In this paper, we carried out a feasibility study of the Bayesian approximation error approach in the context of domain modelling errors in the inverse source problem of the Poisson equation. In particular, we estimated sparse dipole source fields in electroencephalographic imaging using a crude three concentric circle model of the head, instead of the accurate head model, and compensated the related domain errors using the BAE approach. The results of this paper suggest that the BAE approach is able to alleviate the effects of an incorrect domain geometry and structure to varying degrees, depending on the number of sources, their location and the signal to noise ratio of the boundary data.

Another uncertainty that is critical in inverse source problems is the unknown material properties of the domain. We note that the extraction of the accurate material property values is also a challenge, for example, when brain imaging is considered. Therefore, to assess the performance of BAE further, the effect of varying material properties should be considered. 


\section{Acknowledgment}

Dr. Koulouri would like to thank the John S. Latsis Public Benefit Foundation and Dr. Rimpiläinen the Finnish Cultural Foundation for financial support.

\section{References}

\section{References}

[1] S. R. Arridge, J. P. Kaipio, V. Kolehmainen, M. Schweiger, E. Somersalo, T. Tarvainen, M. Vauhkonen, Approximation errors and model reduction with an application in optical diffusion tomography, Inverse Problems 22 (2006) 175-195.

[2] J. P. Ary, S. A. Klein, D. H. Fender, Location of sources of evoked scalp potentials: corrections for skull and scalp thickness, IEEE Trans. Biomed. Eng. 28 (1981) 447-452.

[3] K. Awada, D. Jackson, S. B. Baumann, J. Williams, D. Wilton, P. Fink, B. Prasky, Effect of conductivity uncertainties and modeling errors on EEG source localization using a 2-D model, IEEE Trans. Biomed. Eng. 45 (9) (1998) 1135-1145.

[4] S. Baillet, J. J. Riera, G. Marin, J. F. Mangin, J. Aubert, L. Garnero, Evaluation of inverse methods and head models for EEG source localization using a human skull phantom, Phys. Med. Biol. 46 (2001) 77-96.

[5] S. P. Boyd, L. Vandenberghe, Convex Optimization, Cambridge University Press, 2004.

[6] D. Calvetti, E. Somersalo, Introduction to Bayesian scientific computing: Ten lectures on subjective computing, Springer, 2007.

[7] B. N. Cuffin, Effects of head shape on EEGs and MEGs, IEEE Trans. Biomed. Eng. 37 (1) (1990) 44-52.

[8] B. N. Cuffin, Effects of local variations in skull and scalp thickness on EEG's and MEG's, IEEE Trans. Biomed. Eng. (1) (1993) 42-48. 
[9] L. Ding, B. He, Sparse source imaging in electroencephalography with accurate field modeling, Hum. Brain Mapp. 29 (9) (2008) 1053-1067.

[10] M. L. Eaton, Multivariate Statistics: A Vector Space Approach, Institute of Mathematical Statistics, 2007.

[11] M. Fuchs, J. Kastner, M. Wagner, S. Hawes, J. S. Ebersole, A standardized boundary element method volume conductor model, Clin. Neurophysiol. 113 (5) (2002) 702-712.

[12] R. Grech, T. Cassar, J. Muscat, K. Camilleri, S. Fabri, M. Zervakis, P. Xanthopoulos, V. Sakkalis, B. Vanrumste, Review on solving the inverse problem in EEG source analysis, J. Neuroeng. Rehabil. 5 (1) (2008) 25 .

[13] M. Hämäläinen, R. Hari, R. J. Ilmoniemi, J. Knuutila, O. V. Lounasmaa, Magnetoencephalography - theory, instrumentation, and applications to noninvasive studies on the working human brain, Rev. Mod. Phys. 65 (2) (1993) 413-497.

[14] W. Han, W. Cong, G. Wang, Mathematical theory and numerical analysis of bioluminescence tomography, Inverse Problems 22 (2006) 16591675 .

[15] S. Haufe, V. V. Nikulin, A. Ziehe, K.-R. Müller, G. Nolte, Combining sparsity and rotational invariance in EEG/MEG source reconstruction., NeuroImage 42 (2) (2008) 726-738.

[16] G. Huiskamp, M. Vroeijenstijn, R. van Dijk, G. Wieneke, A. C. van Huffelen, The need for correct realistic geometry in the inverse EEG problem, IEEE Trans. Biomed. Eng. 46 (11) (1999) 1281-1287.

[17] J. Kaipio, V. Kolehmainen, Approximate marginalization over modeling errors and uncertainties in inverse problems, in: P. Damien, N. Polson, D. Stephens (eds.), Bayesian Theory and Applications, Oxford University Press, 2013.

[18] J. Kaipio, E. Somersalo, Statistical inverse problems: Discretization, model reduction and inverse crimes, J. Comput. Appl. Math. 198 (2) (2007) 493-504. 
[19] J. P. Kaipio, E. Somersalo, Statistical and Computational Inverse Problems, Applied Mathematical Series, Springer, 2004.

[20] S.-J. Kim, K. Koh, M. Lustig, S. Boyd, D. Gorinevsky, An interiorpoint method for large-scale 11-regularized least squares, IEEE J. Sel. Top. Signal Process. 1 (4) (2007) 606-617.

[21] T. Köhler, M. Wagner, M. Fuchs, H.-A. Wischmann, R. Drenckhahn, A. Theissen, Depth normalization in meg/eeg current density imaging, in: Engineering in Medicine and Biology Society, 1996. Bridging Disciplines for Biomedicine. Proceedings of the 18th Annual International Conference of the IEEE, vol. 2, 1996.

[22] V. Kolehmainen, S. R. Arridge, J. P. Kaipio, M. Schweiger, E. Somersalo, T. Tarvainen, M. Vauhkonen, Approximation errors and model reduction in optical tomography, in: Int. Conf. IEEE Eng. Med. Biol. Soc., 2006.

[23] V. Kolehmainen, M. Schweiger, I. Nissil, T. Tarvainen, S. R. Arridge, J. P. Kaipio, Approximation errors and model reduction in threedimensional diffuse optical tomography, J. Opt. Soc. Am. A 26 (10) (2009) 2257-2268.

[24] A. Lehikoinen, J. M. J. Huttunen, S. Finsterle, M. B. Kowalsky, J. P. Kaipio, Dynamic inversion for hydrological process monitoring with electrical resistance tomography under model uncertainties, Water Resour. Res. 46 (4) (2010) W04513.

[25] S. Lew, D. D. Sliva, M. Choe, P. E. Grant, Y. Okada, C. H. Wolters, M. S. Hämäläinen, Effects of sutures and fontanels on MEG and EEG source analysis in a realistic infant head model, NeuroImage 76 (2013) $282-293$.

[26] A. Lipponen, A. Seppänen, J. P. Kaipio, Nonstationary approximation error approach to imaging of three-dimensional pipe flow: experimental evaluation,, Meas. Sci. Technol. 22 (10) (2011) 104013.

[27] F. Lucka, S. Pursiainen, M. Burger, C. H. Wolters, Hierarchical Bayesian inference for the EEG inverse problem using realistic FE head models: depth localization and source separation for focal primary currents, NeuroImage 61 (4) (2012) 1364-1382. 
[28] D. S. Marcus, T. H. Wang, J. Parker, J. G. Csernansky, J. C. Morris, R. L. Buckner, Open access series of imaging studies (OASIS): cross-sectional MRI data in young, middle aged, nondemented, and demented older adults, J. Cogn. Neurosci. 19 (9) (2007) 1498-1507, URL: http://www.oasis-brains.org.

[29] M. Mozumder, T. Tarvainen, J. P. Kaipio, S. R. Arridge, V. Kolehmainen, Compensation of modeling errors due to unknown domain boundary in diffuse optical tomography, J. Opt. Soc. Am. A 31 (8) (2014) 1847-1855.

[30] A. Nissinen, L. M. Heikkinen, V. Kolehmainen, J. P. Kaipio, Compensation of errors due to discretization, domain truncation and unknown contact impedances in electrical impedance tomography, Meas. Sci. Technol. 20 (10) (2009) 105504.

[31] A. Nissinen, V. Kolehmainen, J. Kaipio, Compensation of modelling errors due to unknown domain boundary in electrical impedance tomography, IEEE Trans. Med. Imag. 30 (2) (2011) 231-242.

[32] G. Nolte, G. Dassios, Analytic expansion of the EEG lead field for realistic volume conductors, Phys. Med. Biol. 50 (16) (2005) 3807-3823.

[33] P. L. Nunez, R. Srinivasan, Electric Fields of the Brain: The Neurophysics of EEG, Oxford University Press, 2006.

[34] J. O. Ollikainen, M. Vauhkonen, P. A. Karjalainen, J. P. Kaipio, Effects of local skull inhomogeneities on EEG source estimation, Med. Eng. Phys. 21 (3) (1999) 143-154.

[35] R. Oostenveld, P. Fries, E. Maris, J.-M. Schoffelen, FieldTrip: Open source software for advanced analysis of MEG, EEG and invasive electrophysiological data, Comput. Intell. Neurosci. (2011) 156869URL: http://fieldtrip.fcdonders.nl/.

[36] R. D. Pascual-Marqui, D. Lehmann, T. Koenig, K. Kochi, M. C. G. Merlo, D. Hell, M. Koukkou, Low resolution brain electromagnetic tomography (LORETA) functional imaging in acute, neurolepticnaive, first-episode, productive schizophrenia, Psychiatry Researchneuroimaging 90 (1999) 169-179. 
[37] O. Pele, M. Werman, Fast and robust earth mover's distances, in: Proc. IEEE Int. Conf. Comp. Vision, 2009, URL: http://www.cs.huji.ac.il/ ofirpele/FastEMD/code/.

[38] M. Petrou, P. Bosdogianni, Image processing - the fundamentals, John Wiley \& Sons, 1999.

[39] B. Roth, M. Balish, A. Gorbach, S. Sato, How well does a three-sphere model predict positions of dipoles in a realistically shaped head?, Electroenceph. Clin. Neurophysiol. 87 (4) (1993) 175-184.

[40] Y. Rubner, C. Tomasi, L. J. Guibas, The Earth Mover's Distance as a Metric for Image Retrieval, Int. J. Comp. Vision 40 (2) (2000) 99-121.

[41] P. H. Schimpf, C. Ramon, J. Haueisen, Dipole models for the EEG and MEG, IEEE Trans. Biomed. Eng. 49 (5) (2002) 409-418.

[42] P. J. Slomka, R. P. Baum, Multimodality image registration with software: state-of-the-art, Eur. J. Nucl. Med. Mol. Imaging 36 (1) (2009) $44-55$.

[43] A. Tarantola, Inverse problem theory and methods for model parameter estimation, Society for Industrial and Applied Mathematics, 2007.

[44] T. Tarvainen, V. Kolehmainen, J. P. Kaipio, S. R. Arridge, Corrections to linear methods for diffuse optical tomography using approximation error modelling, Biomed. Opt. Express 1 (1) (2010) 209-222.

[45] T. Tarvainen, V. Kolehmainen, A. Pulkkinen, M. Vauhkonen, M. Schweiger, S. R. Arridge, J. P. Kaipio, An approximation error approach for compensating for modelling errors between the radiative transfer equation and the diffusion approximation in diffuse optical tomography, Inverse Problems 26 (1) (2010) 015005.

[46] K. Uutela, M. Hämäläinen, E. Somersalo, Visualization of Magnetoencephalographic Data Using Minimum Current Estimates, NeuroImage 10 (2) (1999) 173-180.

[47] S. P. van den Broek, F. Reinders, M. Donderwinkel, M. J. Peters, Volume conduction effects in EEG and MEG, Electroenceph. Clin. Neurophysiol. 106 (6) (1998) 522-534. 
[48] B. Vanrumste, G. V. Hoey, R. V. de Walle, M. D'Hav, I. Lemahieu, P. Boon, Dipole location errors in electroencephalogram source analysis due to volume conductor model errors, Med. Biol. Eng. Comput. 38 (5) (2000) 528-534.

[49] N. von Ellenrieder, C. H. Muravchik, A. Nehorai, Effects of geometric head model perturbations on the EEG forward and inverse problems, IEEE Trans. Biomed. Eng. 53 (3) (2006) 421-429.

[50] J. Vorwerk, J.-H. Cho, S. Rampp, H. Hamer, T. R. Knösche, C. H. Wolters, A guideline for head volume conductor modeling in EEG and MEG, NeuroImage 100 (2014) 590-607.

[51] C. H. Wolters, A. Anwander, X. Tricoche, D. Weinstein, M. A. Koch, R. S. MacLeod, Influence of tissue conductivity anisotropy on EEG/MEG field and return current computation in a realistic head model: A simulation and visualization study using high-resolution finite element modeling, NeuroImage 30 (2006) 813-826.

[52] C. H. Wolters, L. Grasedyck, W. Hackbusch, Efficient computation of lead field bases and influence matrix for the FEM-based EEG and MEG inverse problem, Inverse Problems 20 (4) (2004) 1099-1116.

[53] D. Yao, Electric potential produced by a dipole in a homogeneous conducting sphere, IEEE Trans. Biomed. Eng. 47 (7) (2000) 964-966.

[54] B. Yvert, O. Bertrand, M. Thvenet, J. F. Echallier, J. Pernier, A systematic evaluation of the spherical model accuracy in EEG dipole localization, Electroenceph. Clin. Neurophysiol. 102 (5) (1997) 452-459.

[55] Z. Zhang, A fast method to compute surface potentials generated by dipoles within multilayer anisotropic spheres, Phys. Med. Biol. 40 (3) (1995) 335-349. 


\section{List of figures}

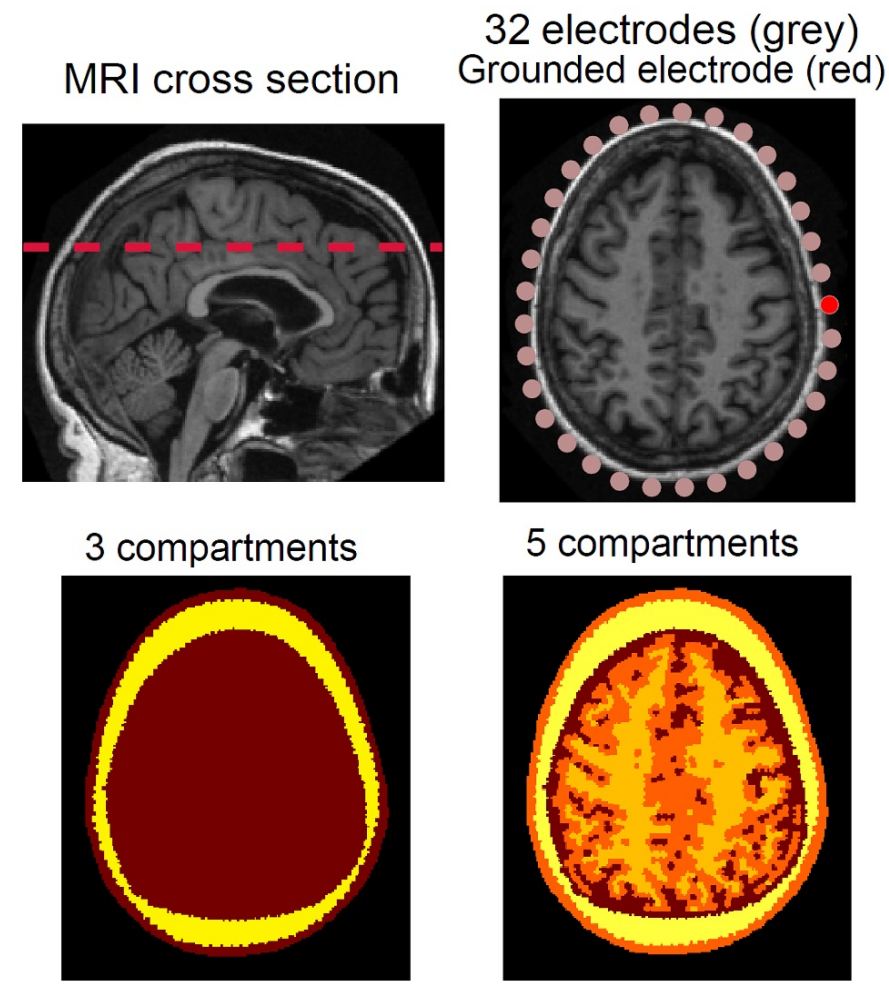

Figure 1: Top left: MRI image of a head with a dashed line that shows the level of the studied cross section. Top right: 32 point electrodes and a ground (above right ear) were equally spaced around the studied cross section. Bottom left: the head cross section is segmented into three compartments: scalp, skull and brain. Bottom right: the head cross section is segmented into five compartments: scalp, skull, cerebral spinal fluid, gray matter and white matter. 


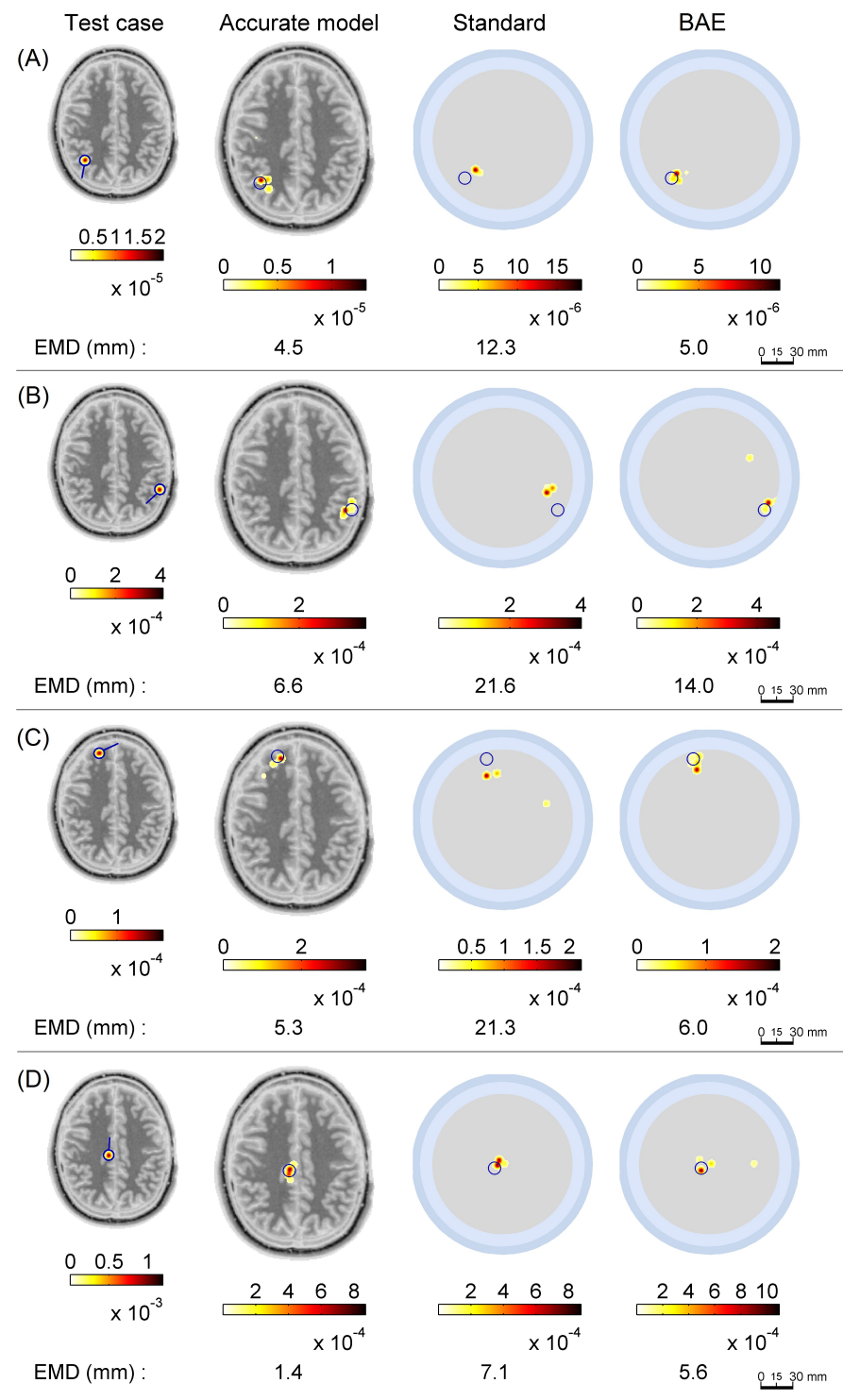

Figure 2: Four test cases with one focal dipole source are shown in the left column, the blue circles with small lines show the locations and orientations of the dipoles. Second column: reconstructions with the accurate model. Third column: the standard (concentric three circle) model. Fourth column: BAE solutions in the same geometry. The earth mover's distances (EMD) are shown below the images. The blue circles show the (projected) locations of the sources. 


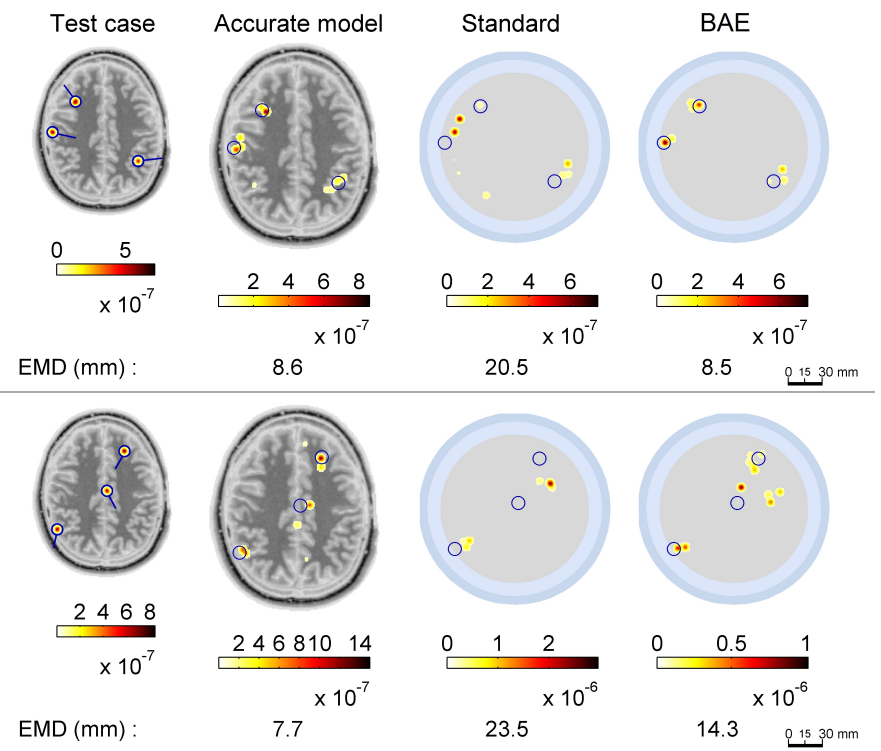

Figure 3: Two test cases with three focal sources are shown on the left. The rest of the columns are as in Figure 2. 


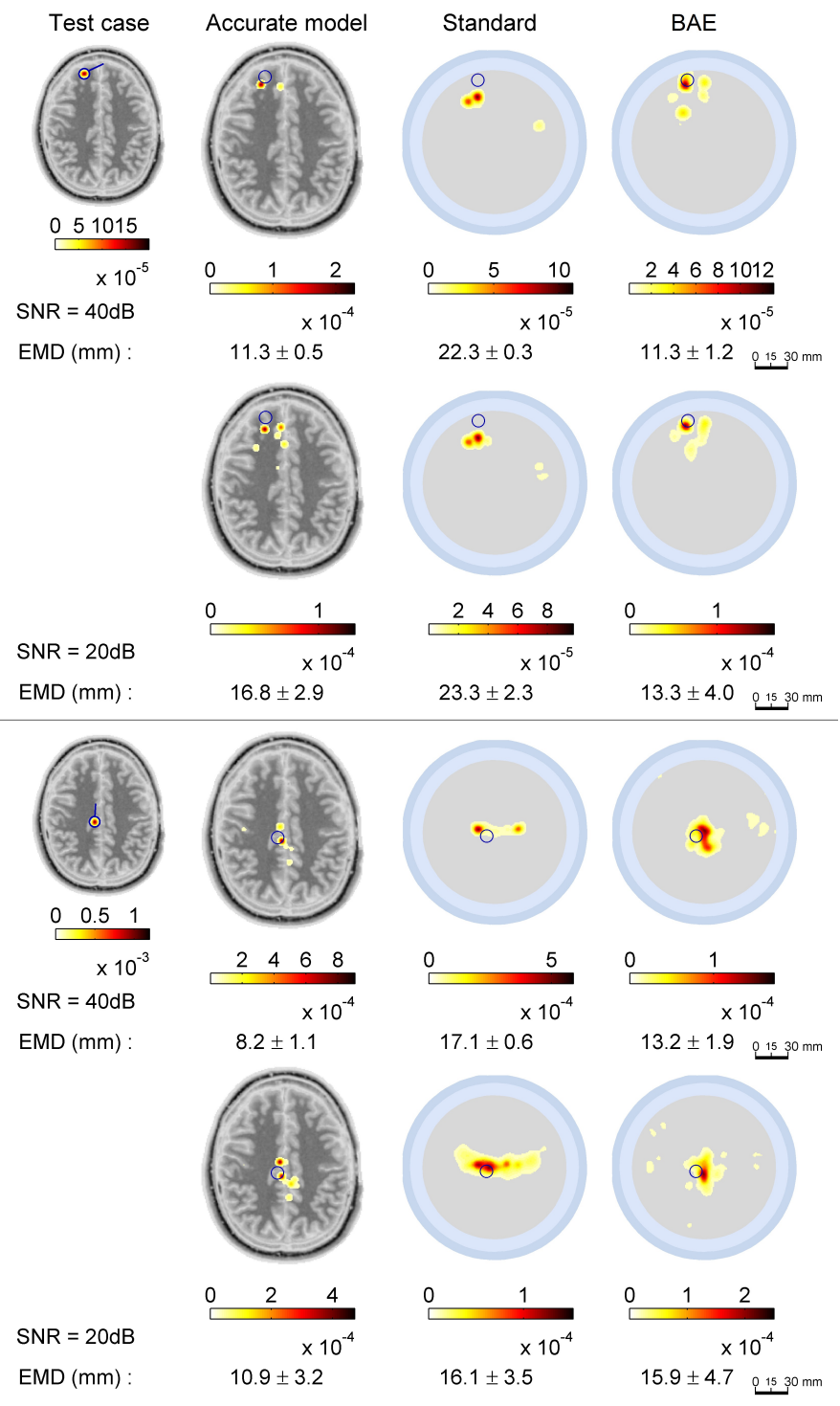

Figure 4: Two test cases with a single focal source are shown on the left, the cases are the same as the ones in Figure $2(\mathrm{C})$ and $(\mathrm{D})$. Here, a five compartment forward model was used and Gaussian white noise was added in the measurements with SNR of $40 \mathrm{~dB}$ and $20 \mathrm{~dB}$. The results are averages of 30 different realizations, and the corresponding averages and standard deviations of the EMD values are below the reconstructions. 


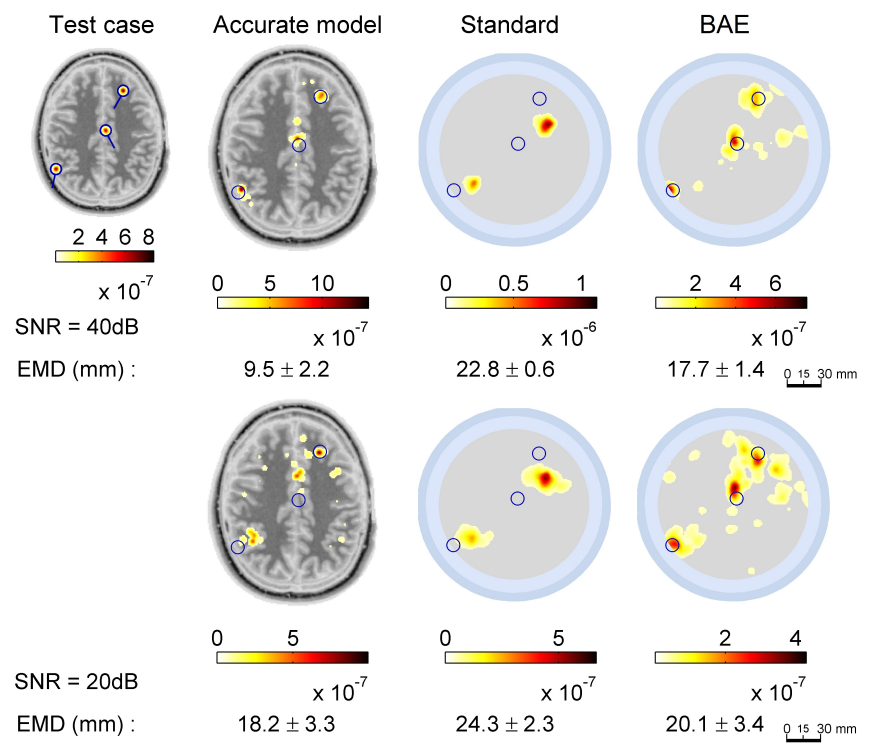

Figure 5: A test case with three focal sources is shown on the left. The other columns show the solutions with the three models as explained in Figure 4. 